\title{
Surface convection in Population II stars
}

\author{
Laurent Piau ${ }^{1}$ and Robert F. Stein ${ }^{2}$ \\ ${ }^{1}$ CEA-Saclay DSM/IRFU/SAp, L'Orme des Merisiers 91191 Gif-sur-Yvette France \\ ${ }^{2}$ Michigan State University, Department of Physics \& Astronomy East Lansing, MI \\ 48824-2320, USA
}

\begin{abstract}
The initial surface abundances of Population II stars have been altered by the interplay between convection, rotational mixing and diffusion. In particular the shallower the outer convection zone the stronger the diffusion impact. We present preliminary results on constraining the extension of the convection zones of Population II stars thanks to 3D hydrodynamical simulations.
\end{abstract}

Keywords. Convection, stars: populations II

\section{STAGGER}

We use the STAGGER code (Stein \& Nordlund 1998) to investigate the convective and the radiative energy transfer from $\sim 0.5 \mathrm{Mm}$ above the photosphere downto $\sim 3.5$ below it. The computational domain extends over 6 by $6 \mathrm{Mm}$ horizontaly. The current grid has 63 points in each direction. We solve the fully compressible equations of hydrodynamics. The specific internal energy and density of the material entering the computational domain from below are adjusted in order to obtain the desired effective temperature in a given gravity field. Because of the cool effective temperatures a significant part of the energy is carried to the surface in form of ionization energy which makes the choice of a realistic equation of state (EoS) important. We use the OPAL2005 EoS for a pure hydrogen/helium mix with $\mathrm{Y}=0.2479$.

The equation of radiative transfer is :

$$
\cos \theta \frac{d I_{\nu}}{\left(\kappa_{\nu}+\sigma_{\nu}\right) \rho d z}=I_{\nu}-B_{\nu}
$$

With $\theta$ the angle from the vertical, $\kappa_{\nu}$ and $\sigma_{\nu}$ respectively the absorption and diffusion coefficients, $\mathrm{I}_{\nu}$ the specific intensity and $\mathrm{B}_{\nu}$ the Planck function.

The equation of radiative heating writes :

$$
Q_{\text {rad }}=4 \pi \rho \int_{0}^{\infty} \kappa_{\nu}\left(J_{\nu}-B_{\nu}\right) d \nu
$$

With $\mathrm{J}_{\nu}$ the mean intensity and $\rho$ the density.

For each cell these equations are solved numerically along one vertical ray and four slanted rays. We use the opacity binning method (Nordlund 1982) which is intended to compute the thermal structure when the medium is neither optically thin nor thick. For some time it was successfully employed in the solar case and recently for other stars (Ludwig et al. 2006).

\section{Method and first comparison}

We determine the required $\log g$ and $\mathrm{T}_{\text {eff }}$ by building stellar models with the stellar evolution code CESAM code (Morel 1997). CESAM utilizes the same composition, EoS and opacities as STAGGER. Our models have $[\mathrm{Fe} / \mathrm{H}]=-3$ and $\mathrm{Y}=0.2479$. 


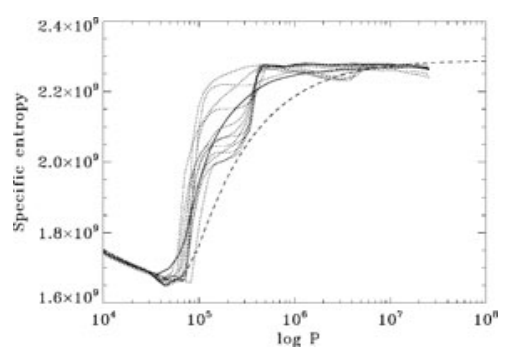

Figure 1. Specific entropy as function of depth (represented by pressure). Black solid line: hydrodynamic model average value. Blue dotted lines spatial fluctuations on the computational box. Green line best fit for a stellar envelope model in terms of $\alpha_{\mathrm{mlt}}$ and for the corresponding $\mathrm{T}_{\text {eff }}=6700 \mathrm{~K}$ and $\log \mathrm{g}=4.2$.

STAGGER was run for 4 hours of surface convection time for surface conditions near the turn-off and and the lower main sequence. ¿From these computations we have inferred

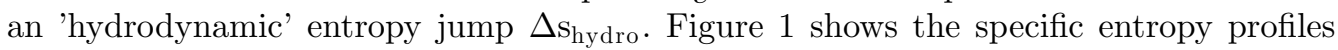
from both hydrodynamical calculations and mixing length theory calculations.

- Near the turn off at $\mathrm{T}_{\text {eff }}=6700 \mathrm{~K}$ and $\log \mathrm{g}=4.2$ we find $\Delta \mathrm{s}_{\text {hydro }}=5.780108 \mathrm{erg} \cdot \mathrm{g}^{-1} \cdot \mathrm{K}^{-1}$.

- Lower on the MS at $\mathrm{T}_{\text {eff }}=5700 \mathrm{~K}$ and $\log \mathrm{g}=4.6$ we find $\Delta \mathrm{s}_{\text {hydro }}=1.702108 \mathrm{erg} \cdot \mathrm{g}^{-1} \cdot \mathrm{K}^{-1}$.

We then buildt grids of envelope model for various $\alpha_{\mathrm{mlt}}, \mathrm{T}_{\mathrm{eff}}$ and $\log \mathrm{g}$ and found the envelope models providing the closest $\Delta \mathrm{s}_{\mathrm{mlt}}$ to $\Delta \mathrm{s}_{\mathrm{hydro}}$ :

- For $\mathrm{T}_{\text {eff }}=6772 \mathrm{~K}$ and $\log \mathrm{g}=4.24$ we have $\Delta \mathrm{s}_{\mathrm{mlt}}=5.782108 \mathrm{erg} \cdot \mathrm{g}^{-1} \cdot \mathrm{K}^{-1}$ when and $\alpha_{\mathrm{mlt}}=1.52$.

- For $\mathrm{T}_{\text {eff }}=5776 \mathrm{~K}$ and $\log \mathrm{g}=4.64$ we have $\Delta \mathrm{s}_{\mathrm{mlt}}=1.705108 \mathrm{erg} \cdot \mathrm{g}^{-1} \cdot \mathrm{K}^{-1}$ when $\alpha_{\mathrm{mlt}}=$ 1.77 .

\section{Conclusion}

- Hydrodynamical 3D simulations can constrain the phenomenological theories used to model convection in stellar evolution. In the context of active research devoted to the oldest stars, we address this issue for extremely metal poor dwarfs.

- As shown by Ludwig et al. (2002) the mixing length theory does not properly describes all the convection properties. Yet obtaining $\alpha_{\text {mlt }}$ thanks to the associated specific entropy jump is sufficient to perform stellar evolution.

- Refined EoS, opacity tables and atmosphere structures are required. The mixing length $\alpha_{\text {mlt }}$ has been constrained for two typical surface conditions of Population II dwarfs in terms of $\mathrm{T}_{\text {eff }}$ and $\log \mathrm{g}$. Once calibrated the mixing length parameters will be used to perform stellar evolution.

\section{References}

Ludwig, H.-G., Allard, F., \& Hauschildt, P. H., 2006, A\&A, 459, 599

Ludwig, H.-G., Allard, F., \& Hauschildt, P. H., 2002, A\&A, 395, 99

Morel, P., 1997, A\&AS, 124, 597

Nordlund, A, 1982, A\&A, 107, 1.

Stein, R. F. \& Nordlund, A, 1998, ApJ, 499, 914 Ferguson, J. W., 2006, ApJ, 653, 300 Chan, K. L., Guenther, D. B., 2003, MNRAS, 340, 923 Franois, P., Bonifacio, P., Barbuy, B., Beers, T., and 4 coauthors, 2005, A\&A, 430, 655 